\title{
THE PECULIAR LIGHT VARIATION OF THE PLANETARY NEBULA NGC 2346
}

\author{
XIANG-LIANG HAO \\ Beijing Astronomical Observatory, Beijing, China
}

\begin{abstract}
Planetary nebula NGC 2346 is a well know butterfly bipolar nebula and the central star (AGK3-O ${ }^{\circ} 965$ ) is known to be a spectroscopic binary with a period of about 16 days $^{(1)}$. This object has been observed frequently and no bright variations were noticed from at least 1899 to $1981 \mathrm{Nov}^{(2)}$. But in 1981 an unexpected large amplitude eclipse light variations were observed by Kohoutek ${ }^{(3)}$. Since then a lot of observations have been reported by many authors. They have revealed that the central star V651 Mon showed fast and complex light variations due to eclipse and the amplitudes of the eclipse varied rapidly.

We have observed this object using the $40 / 200 \mathrm{~cm}$ double astrograph and others at Beijing Observatory since 1981. From these observations we found that its drastic eclipse light variations wich started in 1981, decreased rapidly in 1986 and the eclipse nearly was unseen in the light curves of $1987^{(4)}$.

From the observations during 1988-1991 we found that the brightness variations of V651 Mon have an obvious difference between 1987 and 1988 while we noticed that the eclipse events which nearly unseen in 1987, it reappeared in the light curves of 1988 and its amplitude is about $1.1 \mathrm{mag}$. and the maximum brightness in 1988 were brighter than 1987 and this result were supported by the observations during 1989-1991.

From these it is reasonable to think that this binary central star of planetary nebula NGC 2346 might undergoing another mass ejection from the sdO component during 1987-1988.
\end{abstract}

\section{References}

(1) Mendez, R.H., 1980, IAU Symposium No. 88, p567

(2) Schaefer, B.E., 1983, IBVS, No. 2281

(3) Kohoutek, L., 1982, IBVS, No. 3271

(4) Hao, X-L, 1988, IBVS, No. 3271 